\title{
Time preferences and risk aversion: Tests on domain differences
}

\author{
Christos A. Ioannou ${ }^{1}$. Jana Sadeh $^{1}$
}

Published online: 12 December 2016

() The Author(s) 2016. This article is published with open access at Springerlink.com

\begin{abstract}
Understanding how individuals discount and evaluate the risks of environmental outcomes is a prime component in designing effective environmental policy. We use an incentivized experimental design to investigate whether subjects' time preferences and risk aversion across the monetary and environmental domains differ. We find that subjects' time preferences are not significantly different across the two domains. In contrast, subjects exhibit a higher degree of risk aversion in the environmental domain. Furthermore, we corroborate earlier results, documenting that women are more risk averse than men in the monetary domain, and show this finding to also hold in the environmental domain.
\end{abstract}

Keywords Risk and time · Discounting · Risk aversion - Domain differences · Environmental domain

\footnotetext{
We are grateful to Michael Vlassopoulos, Manos Mentzakis, Zacharias Maniadis and Daniel Read for their comments and suggestions. We would also like to thank Stewart Barr, Andrew Darnton and the Department for Environment, Food \& Rural Affairs for providing the questionnaire and guidance to the methodology. Finally, we are indebted to the Editor-in-Chief, Kip Viscusi, the Editorial Board and an anonymous referee for their detailed and helpful comments, which significantly improved the paper. The research was supported by research funds from the Economic and Social Research Council (ESRC) and the Strategic Research Development Fund (SRDF) of the University of Southampton. The usual disclaimer applies.
}

Electronic supplementary material The online version of this article (doi:10.1007/s11166-016-9245-8) contains supplementary material, which is available to authorized users.

Christos A. Ioannou

C.Ioannou@soton.ac.uk

Jana Sadeh

J.Sadeh@ soton.ac.uk

1 Department of Economics, University of Southampton, Southampton, SO17 1BJ, UK 
JEL Classification C51 $\cdot$ C91 $\cdot$ D03 $\cdot$ Q05

\section{Introduction}

The design and evaluation of environmental policy requires the incorporation of time and risk elements, as many environmental outcomes extend over long time periods and involve a large degree of uncertainty. Understanding how individuals discount and evaluate risks with respect to environmental outcomes is a prime component in designing effective environmental policy to address issues of environmental sustainability, such as climate change. Our objective in this study is to investigate whether subjects' time preferences and risk aversion across the monetary domain and the environmental domain differ.

We elicit subjects' time preferences and risk aversion using a controlled 'withinsubject' experimental design. First, to isolate the effect of domain on intertemporal choices, we use the fixed-sequence choice titration (Harrison and Lau 2005; Read et al. 2005; Andersen et al. 2008; Hardisty and Weber 2009). In this approach, subjects are presented with a series of binary intertemporal choices between a fixed amount that is due at one point in time (henceforth referred to as smaller sooner) and a larger amount that is due at a later point in time (henceforth referred to as larger later). While the smaller sooner amount is kept fixed, the larger later amount increases successively. In the beginning, subjects typically prefer the smaller sooner amount to the larger later one. However, at some point, a switch takes place from the smaller sooner to the larger later amount, which enables the experimenter to extract the discount-rate bracket within which the individual's rate of time preference lies. Second, to elicit subjects' risk aversion, we use a variant of the Eckel-Grossman test (Eckel and Grossman 2002, 2008), where subjects are presented with five gambles of varying riskiness and are required to select the one they prefer. Crucially, in order to ensure that the magnitude of the choices in the monetary domain matched those in the environmental domain, prior to running the experimental sessions, we calibrated the value of the environmental instrument using two contingent valuation studies. Finally, we use the Cognitive Reflection Test and a questionnaire to obtain a measure of subjects' cognitive ability to reflect and deliberate in the face of intuitively simple alternatives as well as insights into subjects' environmental attitudes, which could possibly relate to the way different domains are evaluated.

A novelty of the experimental design is that it is incentivized: in the monetary domain, time preferences and risk aversion are elicited with real monetary payoffs, whereas in the environmental domain, we elicit time preferences and risk aversion using real (bee-friendly) plants. ${ }^{1}$ Our choice for the appropriate environmental instrument was not an easy one. The instrument had to be familiar to subjects to facilitate their understanding of its potential benefits, and credible so that subjects could rest assured that the project is one that can be easily implemented without arousing

\footnotetext{
${ }^{1}$ There exists evidence to suggest that incentivized experiments may have an impact on the discount rates elicited (Coller and Williams 1999; Kirby and Maraković 1995). In fact, Andersen et al. (2014) claim “... the evidence is overwhelming that there can be huge and systematic hypothetical biases" (p. 27).
} 
suspicion of deception. Finally, the instrument had to be divisible in order to enable us to vary the larger later amount and the gambles. The choice of a locally-based project that distributed bee-friendly plants fulfilled all these requirements. ${ }^{2}$

Our first set of main results does not find any significant differences in subjects' time preferences across the monetary and environmental domains. Assuming away any philosophical or ethical issues that might dictate what the discount rate ought to be in environmental cost-benefit analysis, a corollary of the first result is that the same discount rate used for financial payoffs should also be used for the environmental ones when evaluating environmental policies. This corollary is reassuring to economists and policy makers who, for some time now, have been evaluating environmental policies with discount rates that are based on the intertemporal-choice framework of the monetary domain.

Our second set of main results finds domain differences in subjects' risk aversion. More specifically, subjects (men and women) exhibit a higher degree of risk aversion in the environmental domain relative to the monetary domain; that is, individuals tend to be more reluctant to take on large gambles with environmental outcomes than with monetary ones. A plausible explanation for the emergence of domain differences in risk aversion could be stemming from individuals' perception on the consequences of climate change - a topic that has been well publicized. Furthermore, we corroborate existing results, which document that women are more risk averse than men in the monetary domain. We show this finding to also hold in the environmental domain. The latter findings seem to hint that women are more risk averse than men in most domains. In fact, this conjecture finds support in the study of Weber et al. (2002) who show that women are more risk averse than men in four domains (financial decisions, health/safety, recreational, ethical), but not in the domain of social decisions. The authors attribute this pattern of results to gender differences in the perception of risk.

Finally, our third set of results finds no correlation between subjects' time preferences and their risk aversion within a domain. Given that part of the motivation for discounting future outcomes is that an element of risk is introduced by the time delay, one would expect some moderate correlation between the two decision types within a domain. However, this is not the case. Moreover, we do not find any support of the hypothesis that time preferences or risk aversion are correlated with subjects' cognitive abilities or environmental awareness.

The paper adheres to the following plan. We present next an overview of the related literature. Section 3 describes, in relative detail, the experimental design. Section 4 presents the data analysis, and Section 5 discusses the important findings and provides direction for future research.

\section{Literature review}

Our paper is related to two main strands of the literature on decision-making across domains. First, it is related to the growing literature on domain differences in time

\footnotetext{
${ }^{2}$ To the best of our knowledge, this is the first study to use a real environmental instrument; the only other study that we are aware of that investigates differences in time preferences across the monetary and the environmental domains used hypothetical environmental payoffs (Hardisty and Weber 2009).
} 
preferences. The impact of domains on intertemporal choice has predominantly revolved around the monetary domain and the health domain, where most studies find differences in subjects' discounting behavior. ${ }^{3}$ Recently, in the midst of a public debate on the appropriate discount rate to evaluate the consequences of climate change (Stern 2007; Nordhaus 2007), ${ }^{4}$ the investigation on the impact of domains on intertemporal choice has expanded to also include the environmental domain. In their study, Hardisty and Weber (2009) compare intertemporal choices elicited in the three aforementioned domains and find that subjects' discounting behavior is not statistically different across the environmental and monetary domains, but is statistically different across the health domain and the other two domains. Hardisty and Weber (2009) attribute the domain effect in health to subjects' visceral reaction to the health scenarios. Other studies have also assessed the discounting behavior of subjects in the environmental domain albeit via risk assessments. Böhm and Pfister (2005), for example, conduct experiments to measure subjects' risk assessment of hypothetical scenarios on coastal erosion and marine oil spills. The authors find that temporal discounting of environmental risks is weak and postulate that ethical evaluations are not discounted by subjects. Along the same lines, Gattig and Hendrickx (2007) conclude that temporal discounting is less pronounced for environmental risks than for risks in other domains. Finally, Viscusi et al. (2008) estimate discounting rates based on a series of environmental policy choices administered in a survey context. A key finding of their study is that discounting behavior differs markedly for people who visit lakes, rivers and streams for recreational purposes versus those who do not. More specifically, regular visitors to water bodies have low discounting rates, whereas those who do not visit water bodies often have consistently high discounting rates.

Our work is also related to the literature on risk preferences across domains. Weber et al. (2002) look at respondents' choices in various domains and find differences in risk taking across domains. However, the authors attribute these differences to different perceptions of the risks in those domains rather than differences in attitude towards those perceived risks, which they find to be remarkably consistent across domains. In a more recent study, Dohmen et al. (2011) use a large, representative survey of the German population to elicit risk preferences across a number of domains and find that the self-reported, risk-taking measures are highly, but not perfectly, correlated across domains. ${ }^{5}$ Finally, in a study with a different flavor, Riddel (2012) compares subjects' evaluation of financial and environmental lotteries to determine whether preferences over environmental risks can be reasonably approximated by the Expected Utility framework. The author finds that subjects are more

\footnotetext{
${ }^{3}$ Many studies find that discount rates in the health domain are larger than those in the monetary domain for health gains, but lower than those in the monetary domain for health losses (Cairns 1992; Chapman and Elstein 1995; Madden et al. 1999).

${ }^{4}$ As Weitzman (2007) aptly notes, "it is not an exaggeration to say that the biggest uncertainty of all in the economics of climate change is the uncertainty about which interest rate to use for discounting" (p. 705).

${ }^{5}$ Dohmen et al. (2011) test the following domains: career choices, leisure and recreational activities, financial decisions, health and driving. They use a scale from 1 to 10 , with 10 signifying the greatest willingness to take risks.
} 
likely to overemphasise low probability, extreme environmental outcomes than low probability, extreme financial ones. As a result, she concludes that the Expected Utility framework is likely to underestimate subjects' willingness to pay for environmental cleanup programs or policies with uncertain outcomes.

All the aforementioned studies that pertain to the environmental domain use an elicitation method based on hypothetical environmental gains and losses. In sharp contrast, our study uses an incentivized scheme with real monetary and environmental payoffs. The use of real payoffs creates a strong incentive for subjects to display their true preferences and increases the attention given to the task at hand. There is evidence that the use of real payoffs might matter in discounting experiments. For instance, Kirby and Maraković (1995) find that discount rates elicited for real monetary payoffs are higher than those elicited for hypothetical ones. Testing a similar setting, Coller and Williams (1999) are less conclusive in their findings, while Camerer and Hogarth (1999), in a wide survey on the role of real incentives, find no effect on mean performance albeit they find a reduction in variance with high financial incentives. The authors note that high incentives improve performance in demanding tasks and reduce generosity and risk-seeking behavior. The effect of incentives is also confirmed in other studies investigating the impact of real payoffs in experiments (Kroll et al. 1988; Cummings et al. 1995).

\section{Experimental design}

Our experimental setup featured six tasks. Two of these tasks aimed to investigate subjects' intertemporal choices across the monetary domain and the environmental domain. The two tasks differed solely on the instrument that was discounted; that is, the valuation of the two instruments was identical (see Section 3.2). In the monetary domain, the instrument that was discounted was money, whereas in the environmental domain the instrument that was discounted was plants. To isolate the effect of domain on intertemporal choices, we used the fixed-sequence choice titration (Harrison and Lau 2005; Andersen et al. 2008; Hardisty and Weber 2009; Andersen et al. 2014). ${ }^{6}$ In this approach, subjects are presented with a series of binary choices between a fixed amount that is due at one point in time and a larger amount that is due at a later point in time. While the smaller sooner amount is kept fixed, the

\footnotetext{
${ }^{6}$ Other studies (Raineri and Rachlin 1993; Green et al. 1994) have used the staircase choice titration method. The latter method presents subjects with an initial binary intertemporal choice that dynamically adapts the subsequent choices depending on the subject's decisions. Finally, a third method is the matching-tasks method (Kirby and Maraković 1995; Chapman 1996; Cairns and van der Pol 1999), where subjects are asked to indicate what amount they would require in order to postpone the receipt of a given outcome by a given time delay. In essence, this method asks subjects to reveal directly the upper bracket of their indifference point. However, Hardisty et al. (2013) note that choice-based measures, such as the fixed-sequence choice titration and the staircase choice titration, are better predictors of real world outcomes than matching tasks. Additionally, the authors point out that the demanding dynamic staircase titration offers no advantages over the simpler fixed-sequence choice titration, making the latter the most appropriate method for this experiment.
} 
larger later amount increases successively. The experimental data on the repeated binary intertemporal choices are transformed into a single switching point; the latter produces a discount-rate interval, which contains the indifference point of each subject. Another two tasks aimed to measure the risk aversion of subjects across the monetary domain and the environmental domain. The tests on risk aversion were based on the Eckel-Grossman test (Eckel and Grossman 2002, 2008) and differed only on the measurement instrument. Analogous to the previous setup, in the monetary domain, the test used monetary gambles, whereas in the environmental domain, the test used gambles in plants. In addition to the four aforementioned tasks, subjects were required to take the Cognitive Reflection Test (CRT) and to complete a questionnaire. On one hand, the CRT allowed us to obtain a measure of subjects' cognitive ability to reflect and deliberate in the face of intuitively simple alternatives. On the other hand, the questionnaire allowed us to elicit subjects' environmental attitudes. ${ }^{7}$ In summary, the addition of the latter two tasks served to provide insights into possible individual heterogeneity that could be related to the way different domains were evaluated by subjects.

Our experimental design applied a hybrid of a 'within-subject' and 'betweensubject' design (Charness et al. 2012). In line with a standard 'within-subject' design, each subject was exposed sequentially to the six tasks. We safeguarded against the possibility of observing order effects by splitting the sample into four subsamples (A1, A2, B1 and B2). The four subsamples differed only in the order the first four tasks were presented (i.e. the monetary discounting task, the monetary risk aversion test, the environmental discounting task and the environmental risk aversion test), thereby replicating a 'between-design' for these four tasks. This allowed us to harness the strength of each design while safeguarding against possible confounds. The experimental design is indicated in Table 1. In Panel A, we provide a brief description of the task and the corresponding acronym. In Panel B, we display the order of the tasks in the four subsamples.

\subsection{Environmental instrument}

Our choice for the appropriate environmental instrument was not an easy one. We required an instrument that was divisible so we could vary the larger later amount and the gambles. The instrument had to be familiar to subjects to facilitate their understanding of its potential benefits as well as credible so that subjects could rest assured that the project is one that can be easily implemented and would not suspect the experimenter of deception. The choice of a locally-based project that distributed bee-friendly plants fulfilled all these requirements. Subjects were instructed that beefriendly plants would be handed out to staff and students on campus to be placed in outdoor areas. Given the different delay periods, different bee-friendly plants were chosen. Subjects were informed that the plants distributed would be chosen depending on the season to ensure that they are immediately beneficial.

\footnotetext{
${ }^{7}$ The questions were taken from the Segmentation Model created by the Department for Environment, Food \& Rural Affairs DEFRA (2008).
} 
Table 1 Experimental Design

\begin{tabular}{|c|c|c|c|c|}
\hline \multicolumn{5}{|l|}{ Panel A } \\
\hline \multicolumn{3}{|l|}{ Task } & \multicolumn{2}{|c|}{ Acronym } \\
\hline \multicolumn{3}{|l|}{ Monetary Discounting } & \multicolumn{2}{|l|}{ MD } \\
\hline \multicolumn{3}{|l|}{ Monetary Risk Aversion Test } & \multicolumn{2}{|l|}{ MRAT } \\
\hline \multicolumn{3}{|l|}{ Environmental Discounting } & \multicolumn{2}{|l|}{ ED } \\
\hline \multicolumn{3}{|l|}{ Environmental Risk Aversion Test } & \multicolumn{2}{|l|}{ ERAT } \\
\hline \multicolumn{3}{|l|}{ Cognitive Reflection Test } & \multicolumn{2}{|l|}{ CRT } \\
\hline \multicolumn{3}{|l|}{ Questionnaire } & \multicolumn{2}{|l|}{$\mathrm{Q}$} \\
\hline \multicolumn{5}{|l|}{ Panel B } \\
\hline \multicolumn{5}{|c|}{ Subsamples } \\
\hline Stage & $\mathrm{A} 1$ & $\mathrm{~A} 2$ & B1 & B2 \\
\hline 1 & MD & MRAT & ED & ERAT \\
\hline 2 & MRAT & MD & ERAT & ED \\
\hline 3 & $\mathrm{ED}$ & ERAT & MD & MRAT \\
\hline 4 & ERAT & ED & MRAT & MD \\
\hline 5 & CRT & CRT & CRT & CRT \\
\hline 6 & Q & Q & Q & Q \\
\hline \# of Subjects & 31 & 31 & 27 & 29 \\
\hline \# of Sessions & 2 & 2 & 2 & 2 \\
\hline
\end{tabular}

Notes: In Panel A, we provide a brief description of the task and the corresponding acronym. In Panel $\mathrm{B}$, we display the order of the tasks in the four subsamples. The last 2 tasks were common in all four subsamples. The first four tasks (MD, MRAT, ED and ERAT) were shuffled across the four subsamples. The last two rows display the total number of participants and the number of sessions in each subsample

The environmental project was described in a succinct and neutral manner. The link between bee-friendly plants and the positive externality they generate was stated in the description. We also stated the fact that bee populations are in decline. These two facts are central to the positioning of the project as an environmentally beneficial one. A total of 63 plants were distributed in the experiment. The full description of the project is reported in the Online Appendix.

\subsection{Valuation of a bee-friendly plant}

In order to ensure that the magnitude of the choices in the monetary domain matched that of the choices in the environmental domain, prior to the experimental sessions, we calibrated the value of a bee-friendly plant using two contingent valuation studies carried out at the University of Southampton. ${ }^{8}$ Subjects participating in these studies were given the same project description that was used in the experimental sessions.

\footnotetext{
${ }^{8}$ It is well documented in the literature on discounting that small payoffs are discounted more heavily than larger ones. This regularity is referred to as the magnitude effect (Frederick et al. 2002). Our approach minimizes this effect.
} 
Table 2 Valuation of a Bee-Friendly Plant

\begin{tabular}{ll}
\hline Value Presented & Acceptance Rate \\
\hline$£ 0.50$ & $88 \%$ \\
$£ 2.50$ & $60 \%$ \\
$£ 5.00$ & $41 \%$ \\
$£ 10.00$ & $19 \%$ \\
$£ 15.00$ & $6 \%$ \\
\hline
\end{tabular}

Notes: In the first column, the monetary values that were presented to respondents in the second contingent valuation study are displayed. Subjects were presented with only one out of the five possible values. Subjects were asked whether they were willing to pay that particular amount to contribute one extra plant to the project. The sample consisted of 81 subjects who were split between the five values. In the second column, we display the corresponding acceptance percentages; that is, the percentage of subjects who replied that they would be willing to pay that value to contribute one extra plant to the project

Each study consisted of 81 students of the University of Southampton. The first contingent valuation study presented subjects with an open-ended question asking them to indicate their maximum willingness to pay to contribute one extra plant to the project. The purpose of this study was to allow for the calibration of the values to be used in the second study. The median value of subjects' responses was $£ 5$. The top five modal values were utilized in the second contingent valuation study, which presented subjects with only one out of the five possible values. Subjects were asked whether they were willing to pay that particular amount to contribute one extra beefriendly plant to the project. The sample was split between the five values, with 17 subjects responding to the first value of $£ 0.50,15$ subjects responding to the second value of $£ 2.50,17$ subjects responding to the third value of $£ 5.00,16$ subjects responding to the fourth value of $£ 10.00$ and 16 subjects responding to the final value of $£ 15.00$. The different values presented to respondents and the corresponding acceptance percentages are displayed in Table 2 . We found that the mean willingness to pay was approximately $£ 4.98$. ${ }^{9}$ This specific value was close enough to the median response in the open-ended question of the first study. Consequently, we rounded the number to the nearest pound, and implemented a conversion rate of 1 plant $=£ 5$. Subjects were therefore presented with choices starting at $£ 50$ in the monetary domain and 10 plants in the environmental domain. The plants that were distributed were purchased for $£ 3$ to $£ 5$ each.

\subsection{Tasks}

All experimental sessions consisted of six stages with one task in each stage. Subjects were informed of the total number of stages at the start of the experimental session,

\footnotetext{
${ }^{9}$ The mean willingness to pay was estimated using a probit model with the binary response ('yes' or 'no' to the willingness to pay question) as the dependent variable and the monetary value displayed to the subject as the only explanatory variable along with a constant term.
} 
but were introduced to the tasks of the stages as they progressed through the session. The experimental sessions were conducted in the Social Sciences Experimental Lab (SSEL) at the University of Southampton in March and April of 2014. The subjects were recruited from the student population of the University of Southampton using an electronic recruitment system. Subjects were allowed to participate in only one session. A total of 118 students participated in the experiment. The split across gender was almost even: $54 \%$ were men and $46 \%$ were women. The ages ranged from 18 to 28 . The average age was 20 years old. The bigger portion of subjects (around $60 \%$ ) were pursuing an economics degree. Students pursuing a mathematics degree (around $12 \%$ ) also had a large representation in the sample, as well as students pursuing a philosophy degree (around 4\%). The remaining 24\% of the sample were students studying to earn a degree in either English, history, modern languages, music, chemistry, law, health sciences and geography. Ninety-three percent of the subjects were undergraduates; the rest pursued postgraduate studies. Each session had at most 16 subjects (this is the maximum capacity of the lab) and lasted approximately 45 minutes. The minimum number of subjects in a session was 13. The total number of subjects in each subsample and the total number of sessions in each subsample are displayed in the last two rows in Panel B of Table 1. Each participant received $£ 5$ as a participation fee. The experimental codes were programmed using the experimental software z-Tree (Fischbacher 2007). The experimental instructions are provided in the Online Appendix.

\subsubsection{Monetary Discounting (MD) \& Environmental Discounting (ED)}

The Monetary Discounting (MD) task presented subjects with choices between a smaller sooner amount and a larger later amount. The smaller sooner amount was kept fixed at $£ 50$, whereas the larger later amount started at $£ 55$ and progressively increased to $£ 100$ (i.e. $£ 60, £ 65, £ 70, £ 75, £ 100$ ). Subjects were presented with these six choices for three different delay periods: (i) a 3-month delay period, (ii) a 6-month delay period, and (iii) a 12-month delay period. Thus, in total subjects had to respond to $6 \times 3=18$ questions. The implied hyperbolic discount-rate brackets in each of the delay periods became progressively smaller. Note that the implied discount-rate brackets were not provided to subjects. In total, we extracted three monetary discount brackets for each subject. ${ }^{10}$ The binary choices and the implied hyperbolic discountrate brackets are displayed in Panel A of Table 3. To calculate the implied discount rates, we used the hyperbolic formula $\rho=12(F / P-1) / T$, where $\rho$ is the discount rate, $\mathrm{F}$ is the future value, $\mathrm{P}$ is the present value and $\mathrm{T}$ is the time delay (in months) between the present and the future value (Doyle 2013). ${ }^{11}$

\footnotetext{
${ }^{10}$ Around $92 \%$ of subjects had one switching point. Subjects who switched more than once in two or more discounting tasks were excluded from the analysis on time preferences (see Section 4.1.1).

${ }^{11}$ Here, our objective is to investigate subjects' intertemporal choices across the monetary domain and the environmental domain. We remain agnostic as to the actual numerical value of the discount rate. Calculating the actual discount rate is outside the scope of this study. Nevertheless, our qualitative results are robust to consistent changes in the functional form across the two domains.
} 
Table 3 Binary Choices and Implied Discount-Rate Brackets

\section{Panel A}

Monetary Discounting (MD)

\section{Binary Choice}

Smaller sooner

$(£)$

50

50

50

50

50

50

Larger later
$(£)$
55
60
65
70
75
100

Hyperbolic Discount-Rate Brackets

$\begin{array}{lll}\text { 3-month } & \text { 6-month } & \begin{array}{l}\text { 12-month } \\ (\%)\end{array} \\ \text { (\%) } & (\%) \\ /-40 & /-20 & /-10 \\ 40-80 & 20-40 & 10-20 \\ 80-120 & 40-60 & 20-30 \\ 120-160 & 60-80 & 30-40 \\ 160-200 & 80-100 & 40-50 \\ 200-400 & 100-200 & 50-100\end{array}$

\section{Panel B}

Environmental Discounting (ED)

Binary Choice

Smaller sooner

(plants)

Larger later

(plants)

10

10

10

10

10

10
11

12

13

14

15

20
Hyperbolic Discount-Rate Brackets

$\begin{array}{lll}\begin{array}{l}\text { 3-month } \\ (\%)\end{array} & \begin{array}{l}\text { 6-month } \\ (\%)\end{array} & \begin{array}{l}\text { 12-month } \\ (\%)\end{array} \\ /-40 & /-20 & /-10 \\ 40-80 & 20-40 & 10-20 \\ 80-120 & 40-60 & 20-30 \\ 120-160 & 60-80 & 30-40 \\ 160-200 & 80-100 & 40-50 \\ 200-400 & 100-200 & 50-100\end{array}$

Notes: In Panel A, we display the binary choices and the implied hyperbolic discount-rate brackets in the Monetary Discounting (MD) task. In Panel B, we display the binary choices and the implied hyperbolic discount-rate brackets in the Environmental Discounting (ED) task

All the intertemporal choices presented to participants incorporated a front-end delay as is standard practice in many such experimental studies. Rather than giving subjects an earlier option that is payable at the end of the experimental session, discounting experiments typically make use of a front-end delay where the smaller sooner choice is itself delayed by a short time period (Coller and Williams 1999; Andersen et al. 2008). The main advantage of this approach is that the front-end delay safeguards against possible confounding effects caused by any perceived transaction costs being associated with the larger later payment (Harrison and Lau 2005).

The payment method was designed to further reduce any perceived transaction costs. Subjects were given a requisition form at the end of the experimental session, which detailed their payoffs. The requisition form had to be dropped off at the Finance Office (in the School of Social Sciences at the University of Southampton) 
and participants were paid by direct debit by the Finance Office on the date specified on the form. The precise process was explained in the experimental instructions.

In the Environmental Discounting (ED) task, subjects were presented with the same setup as in the MD task; that is, six binary choices were displayed for each of the (three) different delay periods. Analogous to the task above, three environmental discount brackets were obtained for each subject. The only difference between this task and the previous one is that subjects were presented with the environmental instrument (i.e. plants) instead of money. The binary choices presented to subjects and the implied hyperbolic discount-rate brackets are displayed in Panel B of Table 3.

\subsubsection{Monetary Risk Aversion Test (MRAT) \& Environmental Risk Aversion Test (ERAT)}

The two tests served to elicit subjects' risk aversion in the monetary domain and the environmental domain. We used a variant of the Eckel-Grossman test (Eckel and Grossman 2002, 2008), where subjects were presented with five gambles of varying riskiness and were required to select the one they prefer. All gambles had two possible outcomes: Outcome X with 50\% likelihood and Outcome Y with 50\% likelihood; that is, both outcomes were equiprobable. In addition, the expected payoffs were easy to calculate and the increasing variance as the gambles got riskier was significantly large to be noticeable. This test is a simplified version of the one designed by Holt and Laury (2002), but it still elicits sufficient heterogeneity in subjects' responses (Eckel and Grossman 2008).

The Monetary Risk Aversion Test (MRAT) was set at a magnitude level that was comparable to the choices given to subjects in the discounting tasks. The gambles started at an option with identical outcomes (i.e. a gain of £50) and moved to options of increasing variance at the point where the last option's equiprobable outcomes were $£ 5$ and $£ 162.50$. Expected payoffs increased as you moved down the table, so choices further down indicated lower risk aversion. The Environmental Risk Aversion Test (ERAT) was matched in magnitude to the MRAT at the same conversion rate of money per plant ( $£ 5$ per plant) used in the discounting tasks. Analogous to the MRAT, the first option had identical outcomes, whereas the last option's equiprobable outcomes were 1 plant and 33 plants. The lists of gambles presented in the MRAT and the ERAT are displayed in Panel A and Panel B, respectively, in Table 4.

\subsubsection{Cognitive Reflection Test (CRT) \& Questionnaire $(Q)$}

The Cognitive Reflection Test (CRT) was proposed by Frederick (2005) as a way of measuring a specific type of cognitive ability - that of suppressing a spontaneous response in favor of a more deliberately-thought-out one. The CRT consists of 3 questions. In order to successfully complete the CRT subjects were required to question their initial response and devote some cognitive power to realize that it was incorrect 
Table 4 Risk Aversion Tests

\begin{tabular}{|c|c|c|c|}
\hline \multicolumn{4}{|l|}{ Panel A } \\
\hline \multicolumn{4}{|c|}{ Monetary Risk Aversion Test (MRAT) } \\
\hline Option & Outcome & $\begin{array}{l}\text { Payoffs } \\
(£)\end{array}$ & Probability \\
\hline \multirow[t]{2}{*}{1} & $\mathrm{X}$ & 50.00 & $50 \%$ \\
\hline & $\mathrm{Y}$ & 50.00 & $50 \%$ \\
\hline \multirow[t]{2}{*}{2} & $\mathrm{X}$ & 35.00 & $50 \%$ \\
\hline & $\mathrm{Y}$ & 87.50 & $50 \%$ \\
\hline \multirow[t]{2}{*}{3} & $\mathrm{X}$ & 25.00 & $50 \%$ \\
\hline & $\mathrm{Y}$ & 112.50 & $50 \%$ \\
\hline \multirow[t]{2}{*}{4} & $\mathrm{X}$ & 15.00 & $50 \%$ \\
\hline & $\mathrm{Y}$ & 137.50 & $50 \%$ \\
\hline \multirow[t]{2}{*}{5} & $\mathrm{X}$ & 5.00 & $50 \%$ \\
\hline & $\mathrm{Y}$ & 162.50 & $50 \%$ \\
\hline \multicolumn{4}{|l|}{ Panel B } \\
\hline \multicolumn{4}{|c|}{ Environmental Risk Aversion Test (ERAT) } \\
\hline Option & Outcome & $\begin{array}{l}\text { Payoffs } \\
\text { (plants) }\end{array}$ & Probability \\
\hline \multirow[t]{2}{*}{1} & $\mathrm{X}$ & 10 plants & $50 \%$ \\
\hline & $\mathrm{Y}$ & 10 plants & $50 \%$ \\
\hline \multirow[t]{2}{*}{2} & $\mathrm{X}$ & 7 plants & $50 \%$ \\
\hline & $\mathrm{Y}$ & 18 plants & $50 \%$ \\
\hline \multirow[t]{2}{*}{3} & $\mathrm{X}$ & 5 plants & $50 \%$ \\
\hline & $\mathrm{Y}$ & 23 plants & $50 \%$ \\
\hline \multirow[t]{2}{*}{4} & $X$ & 3 plants & $50 \%$ \\
\hline & $\mathrm{Y}$ & 28 plants & $50 \%$ \\
\hline \multirow[t]{2}{*}{5} & $\mathrm{X}$ & 1 plant & $50 \%$ \\
\hline & $\mathrm{Y}$ & 33 plants & $50 \%$ \\
\hline
\end{tabular}

Notes: Panel A displays the Monetary Risk Aversion Test (MRAT). Panel B displays the Environmental Risk Aversion Test (ERAT). Both panels follow the same structure. In the first column, the 5 options available to subjects are listed. In the second column, the possible outcomes of each option are listed: Outcome X or Outcome Y. In the third column, the payoffs associated with each outcome in each option are listed. Note that the inability to express decimals in plants led us to the rounding up of payoffs in this domain. In column four, the probability of that specific outcome occurring is listed

and, consequently, arrive at the correct answer. The inclusion of the CRT task allows us to capture the heterogeneity in subjects' reflective ability. More specifically, cognitive ability could plausibly be increasingly relevant to the evaluation of intertemporal (monetary and environmental) choices and (monetary and environmental) risk aversion. The three CRT questions are included in the Online Appendix.

Finally, in the last stage, we administered the Questionnaire (Q). The questionnaire consisted of questions of socio-demographic nature as well as 17 questions 
taken from the Segmentation Model developed by DEFRA (2008) ${ }^{12}$ The latter part pertained to subjects' values, attitudes and motivations as well as current behaviors and barriers to change. In addition, the questions covered topics such as climate change, recycling, transportation and water use. The $\mathrm{Q}$ was administered in the last stage in order to remove any unintentional impact these questions might have on the environmental intertemporal choices and environmental risk aversion of subjects.

\subsection{Payment mechanism}

The experimental design applied a variant of the random-lottery incentive scheme, where subjects make a number of decisions knowing that, at the end of the experimental session, one of these decisions will be selected for payment. There is a vast literature testing the validity of this payment scheme. Laury (2005) found that subjects do not scale down decisions when they are only being paid for a subset of these decisions. Along the same lines, Cubitt et al. (1998) confirmed that such design does not contaminate elicited preferences. Hey and Lee (2005) showed that subjects separate the various questions and respond to each question individually and in isolation from the rest; thus, incentives are retained. Recently, Andersen et al. (2014) find no evidence that the use of probabilistic payment schemes on discount rates changes behavior relative to that in a fully-paid experiment. An added value of this approach is that it neutralizes the income effect that would otherwise be experienced as subjects progress through the periods. Our approach was to apply a double layered random-lottery incentive payment scheme. More specifically, two subjects in each experimental session were randomly selected to be paid for their choices. The first subject selected was paid for either the choice made in the MD task or the choice made in the ED task, where each task had an equal probability of being selected. Once the domain was selected, one of the 18 questions was drawn and the subject's choice in that question was paid (with money or plants accordingly). The second subject selected was paid for either the choice made in the MRAT or the choice made in the ERAT, where each test had an equal probability of being selected. Once the test was selected, an outcome was drawn (X or Y where each outcome had an equal probability of being selected) and the subject was paid (with money or plants accordingly) based on the gamble chosen.

The random selection was carried out using a bingo machine that was prominently displayed in the lab. Bingo balls were placed on subjects' desks with the terminal ID number on the ball. Subjects placed the balls into the bingo machine themselves at the end of the experimental session and witnessed the random selection. This was

\footnotetext{
${ }^{12}$ The model segments the population into seven behavioral groups using a number of questions on environmental attitudes and the respondents' age. DEFRA has developed this model in order to further advance behavioral change through social marketing strategies that target specific segments of the population. Their objective is to achieve a more environmentally-friendly lifestyle for the public. Segmentation models are a popular way of investigating the behavior of individuals as it pertains to specific functions of their everyday life, such as transportation choices and water consumption. The advantage of the DEFRA model is that it targets attitudes towards many different environmental sectors, thus achieving a classification that captures an individual's overall attitude to issues of an environmental nature (Jesson 2009; Barr et al. 2011).
} 
necessary to ensure complete transparency of the process. However, the choices of the subjects selected were not revealed to the other subjects as that would violate the confidentiality with respect to their earnings.

\subsection{General hypotheses}

Based on the existing literature (Hardisty and Weber 2009), we first hypothesize that the domain has no impact on subjects' intertemporal choices. This hypothesis is tested by comparing the intertemporal choices taken in the monetary domain with those taken in the environmental domain. The first hypothesis is thus formulated as follows.

H1: Subjects' intertemporal choices are the same across the monetary domain and the environmental domain.

In an analogous manner, we hypothesize that subjects' risk aversion is not influenced by the domain. The second hypothesis is stated next.

H2: Subjects' risk aversion is the same across the monetary domain and the environmental domain.

Our last hypothesis is formulated to determine whether there exists some degree of correlation between the two decision types within a domain.

H3: Subjects' intertemporal choices correlate with their risk aversion within a domain.

\section{Results}

The three hypotheses are formally tested next. Each hypothesis is matched with the corresponding result; that is, result $i$ is a report on the test of hypothesis $i$.

\subsection{Descriptive statistics}

\subsubsection{Time preferences}

Recall that subjects had to decide on the switching point in the 3-month delay period, the 6-month delay period, and the 12-month delay period for both the monetary and the environmental domains. Subjects that switched twice within the same time-delay period in two or more discounting tasks were taken out of the data analysis on time preferences. A total of 10 subjects were excluded leaving us with 108 observations.

We next present the switching distribution of subjects in the three delay periods in the monetary domain and in the three delay periods in the environmental domain. This information is displayed in Fig. 1. An individual with a binary choice of 1 in the monetary domain chose the larger later amount of $£ 55$ in lieu of the earlier 


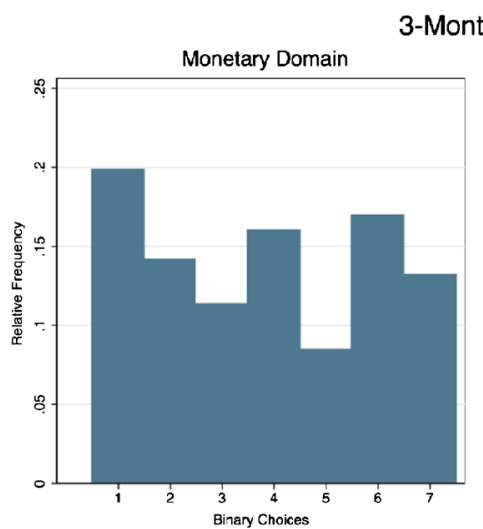

\section{3-Month Delay}

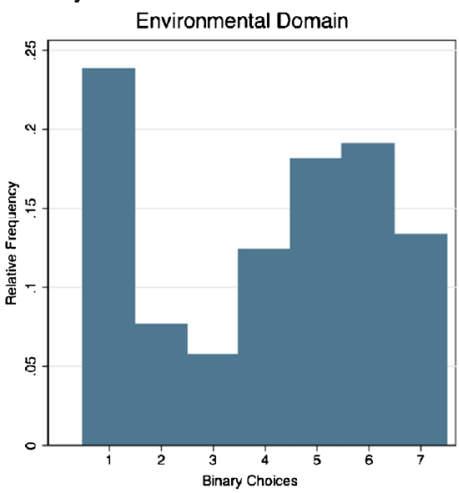

6-Month Delay
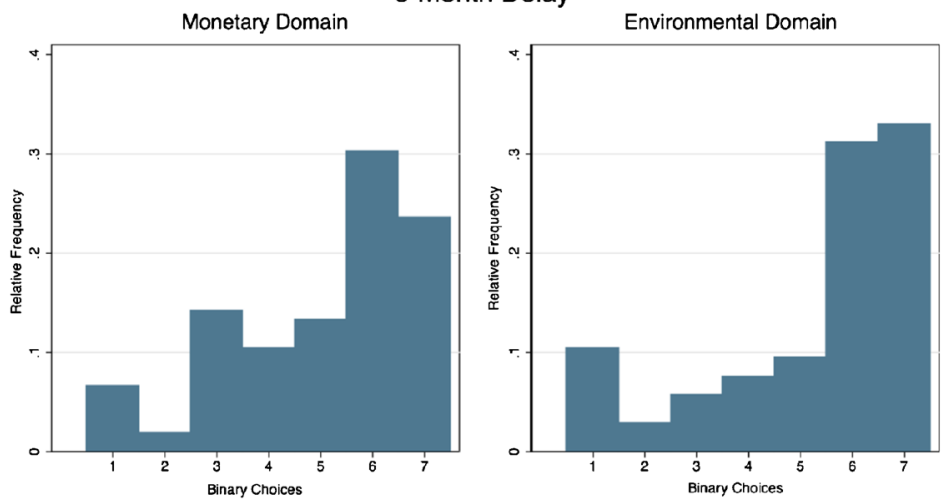

12-Month Delay
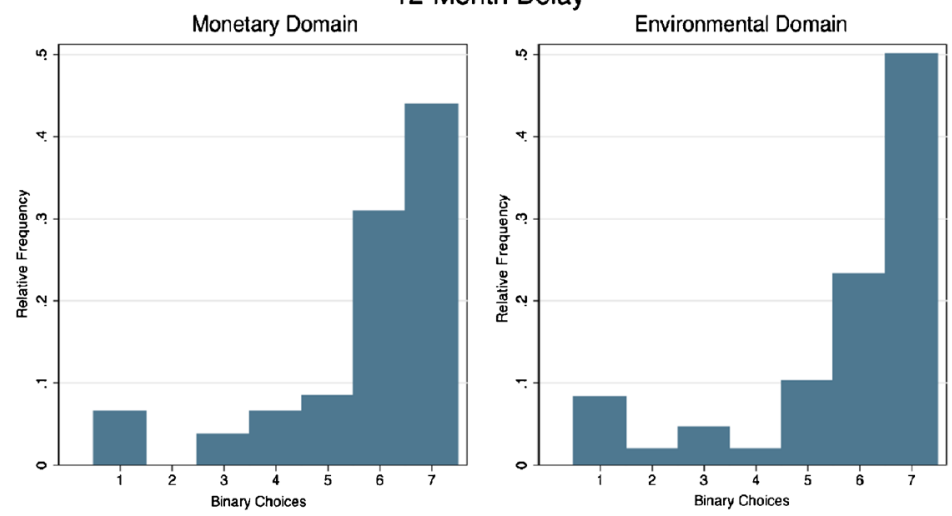

Fig. 1 Switching Distribution. Notes: We display on the left the relative frequency of subjects' switching distributions across the discounting tasks in the monetary domain. We display on the right the relative frequency of subjects' switching distributions across the discounting tasks in the environmental domain. An individual with a binary choice of 1 in the monetary domain chose the larger later amount of $£ 55$ in lieu of the earlier smaller amount of $£ 50$, and an individual with a binary choice of 1 in the environmental domain chose the larger later amount of 11 plants in lieu of the earlier smaller amount of 10 plants. An individual with a binary choice of 7 in either the monetary or the environmental domain always chose the earlier smaller amount 
Table 5 Discounting Patterns Across Domains

\begin{tabular}{lllllll}
\hline Time Delay: & $\begin{array}{l}3 \text {-month } \\
\text { Freq. }\end{array}$ & $\%$ & $\begin{array}{l}\text { 6-month } \\
\text { Freq. }\end{array}$ & $\%$ & Freq. & $\%$ \\
\hline Constant discounting across domains & 27 & 25 & 29 & 27 & 42 & 39 \\
Higher environmental discounting & 46 & 43 & 48 & 44 & 35 & 32 \\
Lower environmental discounting & 35 & 32 & 31 & 29 & 31 & 29 \\
Total & 108 & & 108 & & 108 & \\
\hline
\end{tabular}

Notes: We display information on subjects' discounting behavior in the monetary domain and the environmental domain while controlling for the time delay

smaller amount of $£ 50$, and an individual with a binary choice of 1 in the environmental domain chose the larger later amount of 11 plants in lieu of the earlier smaller amount of 10 plants. An individual with a binary choice of 7 in either the monetary or the environmental domain always chose the earlier smaller amount. The mean switch in the 3-month delay period (MD 3.8/ED 4.0) implies an annual discountrate bracket between $80 \%$ and $160 \%$. The mean switch in the 6-month delay period (MD 5.1/ED 5.3) implies an average discount-rate bracket between $80 \%$ and $200 \%$. Finally, the mean switch in the 12-month delay period (MD 5.5/ED 5.7) implies an average discount-rate bracket between $40 \%$ and $100 \%$.

We next allocate subjects into three categories based on their discounting behavior in the monetary domain and the environmental domain while controlling for the time delay. More specifically, we provide the frequency and percentage of subjects that exhibited one of the three discounting patterns: (i) constant discounting across domains, (ii) higher discounting in the environmental domain, and (iii) lower discounting in the environmental domain. The findings are displayed in Table 5. Over the three time-delay periods, on average, the number of subjects that exhibited a constant discounting behavior across the two domains was $30 \%$. Forty percent of the subjects exhibited a higher discount rate in the environmental domain, and $30 \%$ of the subjects exhibited a lower discount rate in the environmental domain.

\subsubsection{Risk aversion}

In the tests on risk aversion, subjects were given five gambles to choose from, where each gamble featured two possible outcomes: Outcome X with 50\% likelihood and Outcome $\mathrm{Y}$ with $50 \%$ likelihood. The gambles started at a non-degenerate gamble and moved to degenerate gambles of increasing variance and expected payoffs. In the Monetary Risk Aversion Test (MRAT), 18\% of subjects chose the non-degenerate gamble, $59 \%$ of subjects chose one of the next two gambles, while the remaining $23 \%$ chose one of the last two gambles. In the Environmental Risk Aversion Test (ERAT), $28 \%$ of subjects chose the non-degenerate gamble, $54 \%$ of subjects chose one of the next two gambles, and the remaining $18 \%$ chose one of the last two gambles. The 

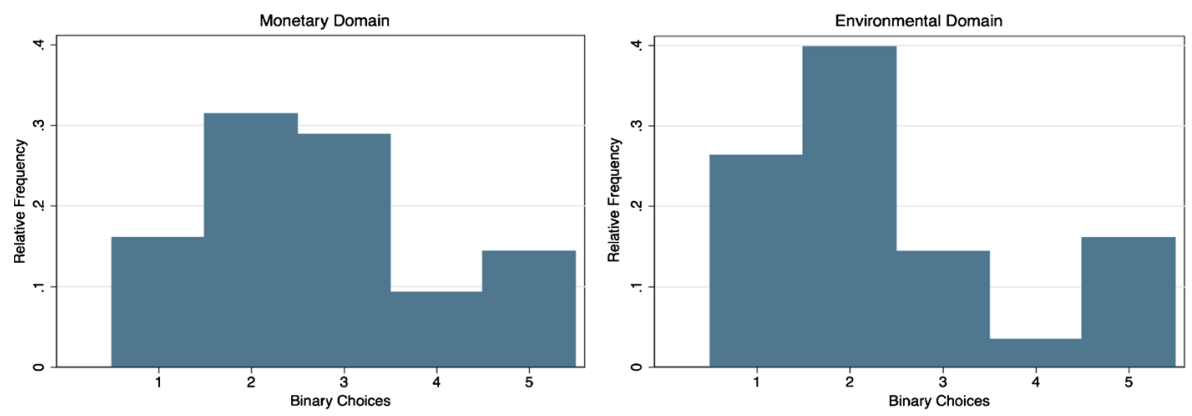

Fig. 2 Distribution of Risk Aversion. Notes: We display on the left the relative frequency of subjects' gambles across the MRAT. We display on the right the relative frequency of subjects' gambles across the ERAT. Binary choices indicate the gambles chosen by the subjects

relative frequency of subjects' gambles across the MRAT and ERAT is displayed in Fig. 2.

\subsubsection{Cognitive Reflection Test (CRT) \& Questionnaire $(Q)$}

The Cognitive Reflection Test (CRT) aims to measure subjects' reflective ability. The test requires subjects to answer three questions, where each question has 4 possible answers. The CRT score consists of one positive point for every correct answer given. The score therefore ranges from 0 to 3 . Thirty-four percent of the subjects answered all three questions correctly, while $18 \%$ of subjects got all three questions wrong. The spread of scores suggests a dispersion of reflective ability amongst subjects. The distribution for the CRT is shown in Fig. 3.

The questionnaire consisted of socio-demographic questions as well as 17 questions taken from the Segmentation Model developed by DEFRA (2008). The questions from the Segmentation Model are provided in Table 6. The model segments the respondents into seven behavioral groups. The frequencies and corresponding percentages of the seven groups are displayed in Table 7 . The first four groups are considered pro-environmental. In our sample, almost $78 \%$ of the respondents belong to one of the top four pro-environmental groups. We further classify subjects that belong to the top four groups as exhibiting environmental awareness.

\subsection{Order effects}

The principal drawback of a 'within-subject' experimental design is the possibility that the order in which the subjects are presented with the tasks might influence their choices. Recall that in our setup, we allowed for four subsamples: A1, A2, B1 and B2 (see Table 1). In Table 8, we use the Bonferroni adjustment, which corrects for multiple comparisons in the $p$-values to determine whether subjects' choice in a specific task differs $(i \neq j)$ across the pairwise, subsample comparison. Rejecting the null would imply that the setup is confounded with order effects. Yet, our design 
Table 6 Segmentation model

Questions

I would only travel by bus if I had no other choice. (PG:3.25; WW:5.33; CC:2.91; SS:6.23; CP:4.14; StSt:5.08; HD:5.48)

For the sake of the environment, car users should pay higher taxes. (PG:5.33; WW:1.87; CC:3.07; SS:3.00; CP:4.90; StSt:3.43; HD:2.62)

People who fly should bear the cost of the environmental damage that air travel causes. (PG:7.44; WW:3.51; CC:4.63; SS:5.15; CP:6.84; StSt:4.70; HD:3.13)

I don't pay much attention to the amount of water I use. (PG:5.11; WW:4.71; CC:5.48; SS:11.22; CP:6.04; StSt:10.01; HD:8.59)

People have a duty to recycle. (PG:17.90; WW:17.37; CC:16.80; SS:17.25; CP:17.03; StSt:17.87; HD:13.30)

We are close to the limit of the number of people that earth can support. (PG:5.23; WW:5.91; CC:2.70; SS:5.60; CP:5.13; StSt:6.64; HD:4.48)

The earth has very limited room and resources. (PG:10.90; WW:9.28; CC:6.73; SS:9.77; CP:8.58; StSt:8.94; HD:7.09)

If things continue on their current course, we will soon experience a major environmental disaster. (PG:15.70; WW:13.51; CC:13.19; SS:15.31; CP:14.75; StSt:16.59; HD:11.19)

The so-called 'environmental crisis' facing humanity has been greatly exaggerated. (PG:7.18; WW:11.42; CC:9.54; SS:8.91; CP:9.77; StSt:12.98; HD:11.94)

It would embarrass me if my friends thought my lifestyle was purposefully environmentally friendly. (PG:1.67; WW:3.23; CC:3.24; SS:3.74; CP:6.06; StSt:8.55; HD:5.34)

Being green is an alternative lifestyle, it's not for the majority. (PG:2.71; WW:4.71; CC:4.27; SS:6.24; CP:5.56; StSt:8.12; HD:6.61)

I find it hard to change my habits to be more environmentally friendly. (PG:6.77; WW:7.12; CC:7.03; SS:9.47; CP:9.60; StSt:11.89; HD:9.77)

It's only worth doing environmentally-friendly things if they save you money. (PG:3.02; WW:4.54; CC:4.93; SS:4.72; CP:6.73; StSt:11.29; HD:7.84)

The effects of climate change are too far in the future to really worry me. (PG:0.46; WW:3.17; CC:2.80; $\mathrm{SS}: 3.56$; CP:4.03; StSt:9.34; HD:6.87)

It's not worth me doing things to help the environment if others don't do the same. (PG:1.19; WW:2.71; CC:2.66; SS:3.42; CP:5.91; StSt:8.09; HD:6.58)

It's not worth Britain trying to combat climate change, other countries will just cancel what we do. (PG:1.18; WW:5.94; CC:1.70; SS:3.53; CP:5.25; StSt:6.21; HD:5.33)

Which of these best describes how you feel about your current lifestyle and the environment? (PG:7.41; WW:3.01; CC:5.56; SS:5.44; CP:6.19; StSt:3.37; HD:2.53)

Notes: We display the questions of the Segmentation Model that classifies respondents into Positive Greens (PG), Waste Watchers (WW), Concerned Consumers (CC), Sideline Supporters (SS), Cautious Participants (CP), Stalled Starters (StSt) and Honestly Disengaged (HD). Each respondent receives 7 scores, one for each behavioral group. The respondent is placed in the group with the highest score. The score of the group is calculated as follows: (i) multiply the unique group coefficient of each question (indicated in brackets) with a scale from 0 to 1 based on the respondent's corresponding answer ('Strongly agree' $=1$, 'Tend to agree' $=0.75$, 'Neither agree nor disagree' $=0.5$, 'Don't know' $=0.25$, and 'Strongly disagree' $=0$; in the last question, the possible responses were: 'I'd like to do a lot more to help the environment' $=1$, 'I'd like to do a bit more to help the environment' $=0.5$, 'I'm happy with what I do at the moment' $=0$, and 'Don't know' $=0.5$ ), (ii) sum the group's products of the 17 questions, (iii) add to the sum in (ii) the product of the respondent's age $\left(16-29=1,30-40=2,41-54=3,55-64=4\right.$, and $\left.65^{+}=5\right)$ and the group's coefficient (PG:1.70, WW:1.75, CC:1.48, SS:1.64, CP:1.53, StSt:1.62, HD:1.50), and (iv) add to (iii) the group's constant (PG:-35.32, WW:-34.28; CC:-26.89, SS:-40.44, CP:-40.02, StSt:-56.70, HD:-35.45) 
Table 7 Behavioral groups

\begin{tabular}{lcr}
\hline Groups & Freq. & $\%$ \\
\hline Positive Greens & 39 & 33.1 \\
Waste Watchers & 6 & 5.1 \\
Concerned Consumers & 30 & 25.4 \\
Sideline Supporters & 16 & 13.6 \\
Cautious Participants & 12 & 10.2 \\
Stalled Starters & 6 & 5.1 \\
Honestly Disengaged & 9 & 7.6 \\
\hline
\end{tabular}

Notes: We classify the 118 respondents into 7 behavioral groups based on the Segmentation Model developed by DEFRA (2008)

does not seem to be susceptible to order effects. Consistency checks using the Šidák and the Scheffé adjustments yield similar results.

\subsection{Domain differences}

The first hypothesis aims to determine whether a change in domain has an effect on subjects' intertemporal choices. We first test our hypothesis using a standard $\chi^{2}$ test, where the $H_{0}$ states that the intertemporal choices across the monetary and the environmental domains are similar when controlling for the time delay. The results are displayed in Table 9, where we report the $p$-values in the full sample and each

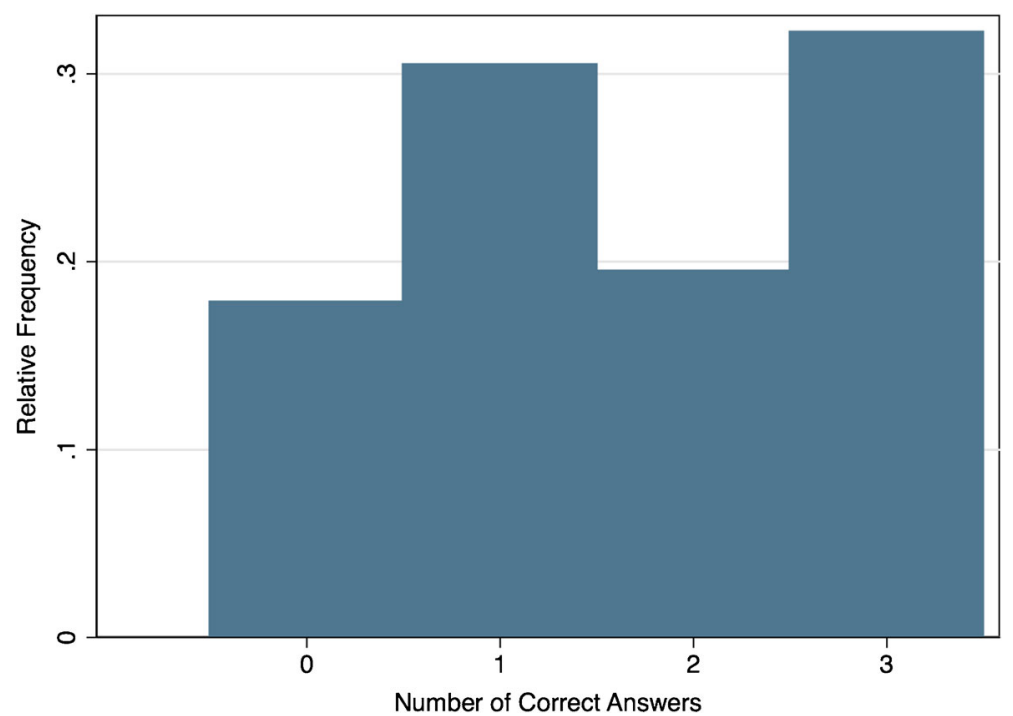

Fig. 3 Distribution of CRT Scores. Notes: We report the relative frequency of subjects' CRT scores. The CRT score consists of one positive point for every correct answer given 
Table 8 Order effects

\begin{tabular}{|c|c|c|c|c|c|c|}
\hline Subsamples & A2/A1 & B1/A2 & B1/A1 & $\mathrm{B} 2 / \mathrm{B} 1$ & B2/A2 & $\mathrm{B} 2 / \mathrm{A} 1$ \\
\hline \multirow[t]{2}{*}{ Alternative hypothesis: } & & & \multicolumn{3}{|c|}{ choice $_{i} \neq$ choice $_{j}$} & \\
\hline & & & \multicolumn{3}{|c|}{$p$-values } & \\
\hline \multicolumn{7}{|l|}{ Time Preferences } \\
\hline MD (3-month delay) & 1.000 & 1.000 & 1.000 & 1.000 & 0.196 & 0.083 \\
\hline MD (6-month delay) & 1.000 & 1.000 & 1.000 & 1.000 & 0.511 & 0.326 \\
\hline MD (12-month delay) & 1.000 & 1.000 & 1.000 & 1.000 & 1.000 & 0.625 \\
\hline ED (3-month delay) & 0.781 & 1.000 & 1.000 & 0.869 & 0.264 & 0.251 \\
\hline ED (6-month delay) & 1.000 & 1.000 & 1.000 & 1.000 & 1.000 & 0.165 \\
\hline ED (12-month delay) & 1.000 & 1.000 & 1.000 & 1.000 & 0.429 & 0.240 \\
\hline \multicolumn{7}{|l|}{ Risk Aversion } \\
\hline MRAT & 0.910 & 1.000 & 1.000 & 1.000 & 1.000 & 1.000 \\
\hline ERAT & 1.000 & 1.000 & 1.000 & 1.000 & 0.413 & 0.145 \\
\hline
\end{tabular}

Notes: We use the Bonferroni adjustment, which corrects for multiple comparisons in the $p$-values to determine whether subjects' choice in a specific task differs $(i \neq j)$ across the pairwise, subsample comparison. MD is the acronym for Monetary Discounting, ED for Environmental Discounting, MRAT for Monetary Risk Aversion Test, and ERAT for Environmental Risk Aversion Test

subsample. The $\chi^{2}$-test does not find any significant differences in discounting across domains; thus, we cannot reject the $H_{0}$.

Moreover, given the repeated nature of the tasks undertaken, we also run two mixed-effects ordered probit regressions in Table 10 with subjects' intertemporal choices as the categorical dependent variable. For the probit regressions, we utilized the full sample as such regressions require a sufficiently large sample size; otherwise the statistical power of the test is significantly compromised. Domain is an explanatory dummy regression variable, which takes the value of 1 if the risk aversion has been obtained from the ERAT and 0 if the risk aversion has been obtained from the MRAT. We investigate two specifications: Model 1 and Model 2. Model 1 incorporates only the domain dummy. Model 2 builds upon the first model

Table $9 \chi^{2}$-tests on domain differences in time preferences

\begin{tabular}{llcccr}
\hline $\begin{array}{l}\text { Subsamples } \\
\text { Alternative hypothesis: }\end{array}$ & All & A1 & A2 & B1 & B2 \\
& & & MD $\neq$ ED & \\
\cline { 3 - 5 } & & & $p$-values & \\
3-month & 0.192 & 0.100 & 0.404 & 0.354 & 0.442 \\
6-month & 0.256 & 0.728 & 0.888 & 0.346 & 0.164 \\
12-month & 0.328 & 0.222 & 0.403 & 0.802 & 0.359 \\
\hline
\end{tabular}

Notes: We report $p$-values from the $\chi^{2}$-test in the full sample and each subsample, where the $H_{0}$ states that the discounting behavior across the across the monetary (MD) and the environmental domains (ED) is similar when controlling for the time delay. 
Table 10 Mixed-effects ordered probit results on domain differences in time preferences

\begin{tabular}{llc}
\hline Variables & Model 1 & Model 2 \\
\hline Domain & 0.106 & 0.189 \\
& $(0.087)$ & $(0.119)$ \\
Gender & & -0.110 \\
& & $(0.203)$ \\
Domain $\times$ Gender & -0.181 \\
& & $(0.174)$ \\
Own home & -0.125 \\
Environmental awareness & $(0.234)$ \\
High CRT & -0.322 \\
& & $(0.214)$ \\
\end{tabular}

Notes: A subject's intertemporal choice is the categorical dependent variable. The two models vary in the number of explanatory variables included. 'Domain' is a dummy that takes the value of 1 in the environmental domain and 0 in the monetary domain, 'Gender' is a dummy that takes the value of 1 if the subject is female and 0 otherwise, 'Domain $\times$ Gender' is an interaction variable for the previous two dummies, 'Own home' is a dummy that takes the value of 1 if the subject's parents own their home and 0 otherwise, 'Environmental awareness' is a dummy that takes the value of 1 if the subject belongs in one of the top four, pro-environmental groups of DEFRA (2008) and 0 otherwise, and 'High CRT' is a dummy that takes the value of 1 if the subject scored at least 2 questions correctly on the CRT and 0 otherwise. All standard errors are reported in parentheses. * Significant at the $10 \%$ level ** Significant at the $5 \%$ level *** Significant at the $1 \%$ level

by adding a gender variable, an interaction variable between gender and domain, a variable on whether the subject's parents own their home, a variable on whether the subject belongs in one of the top four, pro-environmental groups of DEFRA (2008) (i.e. exhibits environmental awareness), and a variable on whether the subject scored at least 2 questions correctly on the CRT. The earlier findings are confirmed; that is, no significant differences in subjects' intertemporal choices seem to exist across the monetary and the environmental domains. Our findings are formalized next in our first main result. ${ }^{13}$

R1: Subjects' intertemporal choices are the same across the monetary and the environmental domains.

\footnotetext{
${ }^{13}$ Recall that the experimental data on the repeated binary intertemporal choices are transformed into a single switching point; the latter produces a discount-rate interval, which contains the indifference point of each subject. Given that the data is of an interval nature and this feature is not captured by the above test, we also run an interval regression, which allows for the specification of the (discount-rate) brackets presented to participants as the dependent variable. More specifically, it allows for the first and last brackets to be open; therefore, the first bracket has no minimum value and the last bracket has no maximum value. The interval regression is run on the log of the discount rates with domain as an explanatory dummy variable, which takes the value of 1 for discounting in the environmental domain and 0 otherwise. The latter model confirms the aforementioned main result.
} 
Table $11 \chi^{2}$-test on domain differences in risk aversion

\begin{tabular}{llcccr}
\hline Subsamples & All & A1 & A2 & B1 & B2 \\
Alternative hypothesis: & & MRAT $\neq$ ERAT & \\
\cline { 3 - 4 } & & $p$-values & & \\
& 0.011 & 0.070 & 0.438 & 0.328 & 0.037 \\
\hline
\end{tabular}

Notes: We utilize the $\chi^{2}$-test in the full sample and each subsample to determine whether subjects' choices on gambles are the same across the two domains

The second hypothesis aims to determine whether a change in domain has an impact on subjects' risk aversion. We thus run a standard $\chi^{2}$-test to examine whether subjects' choices on gambles are the same across domains using the full sample as well as each subsample. Table 11 shows the results of the test. In two of the four subsamples (A1 and B2), there exists evidence to suggest of a domain effect in subjects' choices on gambles. In the full sample, we see that the $H_{0}$ is rejected at the $5 \%$ level of statistical significance; thus, there exists a domain effect on subjects' risk aversion.

In addition, analogous to the aforementioned analysis, we also run two mixedeffects ordered probit regressions in Table 12 with subjects' choices in the tests on risk aversion as the categorical dependent variable. Crucially, we find that subjects exhibit a higher degree of risk aversion in the environmental domain relative to the monetary domain. ${ }^{14}$ These findings culminate in our second main result.

R2: Subjects' risk aversion is statistically different across the monetary and the environmental domains. Specifically, subjects exhibit higher levels of risk aversion in the environmental domain.

Furthermore, it is important to observe that in Model 2 of Table 12, the domain and gender regressors are both significant, while the interaction regressor (domain $\times$ gender) is not. This implies that both men and women exhibit higher levels of risk aversion in the environmental domain than in the monetary one, and that women exhibit higher levels of risk aversion than men in both the monetary and the environmental domains. The finding that women are more risk averse than men in the monetary domain corroborates existing results due to Eckel and Grossman (2002). Crucially, we show this finding to also hold in the environmental domain. Finally, we find that neither time preferences nor risk aversion is correlated with CRT or environmental awareness across the two domains. ${ }^{15}$

\subsection{Time preferences $\&$ risk aversion}

Part of the motivation for discounting future outcomes rests on the element of risk introduced by the time delay. It is possible that time and risk preferences are driven

\footnotetext{
${ }^{14}$ A negative coefficient indicates an increase in the likelihood that a subject will choose one of the earlier (safer) gambles, thereby displaying a higher degree of risk aversion.

${ }^{15}$ This is a departure from the findings of Frederick (2005). We conjecture that differences in the experimental design (Frederick's design was not incentivized) can account for the divergence.
} 
Table 12 Mixed-effects ordered probit results on domain differences in risk aversion

\begin{tabular}{lcc}
\hline Variables & Model 1 & Model 2 \\
\hline Domain & $-0.403^{* * * *}$ & $-0.381^{*}$ \\
& $(0.148)$ & $(0.200)$ \\
Gender & & $-0.582^{* *}$ \\
& & $(0.272)$ \\
Domain $\times$ Gender & -0.050 \\
& & $(0.289)$ \\
Own home & 0.065 \\
& & $(0.288)$ \\
Environmental awareness & -0.199 \\
High CRT & $(0.263)$ \\
& -0.215 \\
\end{tabular}

Notes: A subject's choice in the risk aversion task is the categorical dependent variable. The two models vary in the number of explanatory variables included. 'Domain' is a dummy that takes the value of 1 in the environmental domain and 0 in the monetary domain, 'Gender' is a dummy that takes the value of 1 if the subject is female and 0 otherwise, 'Domain $\times$ Gender' is an interaction variable for the previous two dummies, 'Own home' is a dummy that takes the value of 1 if the subject's parents own their home and 0 otherwise, 'Environmental awareness' is a dummy that takes the value of 1 if the subject belongs in one of the top four, pro-environmental groups of DEFRA (2008) and 0 otherwise, and 'High CRT' is a dummy that takes the value of 1 if the subject scored at least 2 questions correctly on the CRT and 0 otherwise. All standard errors are reported in parentheses. * Significant at the $10 \%$ level ** Significant at the 5\% level *** Significant at the $1 \%$ level

by similar processes. Ferecatu and Öncüler (2016) find evidence that the more risk averse subjects were also more patient. Therefore, we examine next whether the two decision types are correlated within a domain. The mixed-effects ordered probit regression is displayed in Table 13. A subject's choice in the risk aversion task is the categorical dependent variable. The explanatory variables are the switches of the three delay periods; that is, the 3-month, 6-month, and 12-month. We find no significant correlation between the risk aversion variable and any of the discounting variables. This implies that there is no evidence of an individual's intertemporal choices being related to their choices on risk aversion, which culminates in our last main result.

R3: Subjects' intertemporal choices show no correlation with their risk aversion within a domain.

\section{Concluding remarks}

We study experimentally subjects' time preferences and risk aversion across two domains: the monetary domain and the environmental domain. Our study is the first to utilize an incentivized experimental design: in the monetary domain, time 
Table 13 Mixed-effects ordered probit results on time preferences \& risk aversion

\begin{tabular}{lr}
\hline Variables & Model 1 \\
\hline 3-month switch & 0.001 \\
6-month switch & $(0.071)$ \\
12-month switch & 0.057 \\
& $(0.095)$ \\
& -0.022 \\
\hline
\end{tabular}

Notes: A subject's choice in the risk aversion task is the categorical dependent variable. The 3-month switch is a categorical variable representing the choice made in the 3-month delay period. The 6-month switch is a categorical variable representing the choice made in the 6-month delay period. The 12-month switch is a categorical variable representing the choice made in the 12-month delay period. All standard errors are reported in parentheses

preferences and risk aversion are elicited with real monetary payoffs, whereas in the environmental domain, time preferences and risk aversion are elicited using real, bee-friendly plants. Contrasting subjects' intertemporal choices across the monetary and environmental domains, we find that subjects' discounting behavior is not statistically different. In sharp contrast, subjects' risk aversion is significantly different across the monetary domain and the environmental domain; specifically, subjects tend to be unwilling to take on large gambles when it comes to bee-friendly plants. This result is not gender-specific; that is, both men and women exhibit a higher degree of risk aversion in the environmental domain relative to the monetary domain. Moreover, we find that women are more risk averse than men in both the monetary and the environmental domains. Finally, given that part of the motivation to discount future outcomes stems from an element of risk, which is introduced by the time delay, we hypothesize that subjects' intertemporal choices correlate with their risk aversion within a domain. Our analysis reveals no such correlation within a domain.

Ideally, these results ought to be evaluated across three important dimensions. First and foremost, the study should be replicated in a more representative sample given that the present sample consisted only of university students. Second, time preferences and risk aversion in the environmental domain should be tested using other environmental instruments and compared to time preferences and risk aversion in the monetary domain to determine the robustness of the aforementioned findings. For instance, it would be interesting to include instruments that are closer to resembling private goods, such as energy-saving light bulbs or even instruments that confer little private benefit to the recipient, such as supporting endangered species. Third, time preferences and risk aversion should be tested across a much broader array of domains to identify domain-specificity where such exists. Such fruitful attempts have been undertaken in the recent studies of Tsukayama and Duckworth (2010); Einav et al. (2012), and Ubfal (2016). The current research highlights that a direct mapping of results from the monetary domain to the environmental domain is risky. We believe the same holds true for other domains. 
Open Access This article is distributed under the terms of the Creative Commons Attribution 4.0 International License (http://creativecommons.org/licenses/by/4.0/), which permits unrestricted use, distribution, and reproduction in any medium, provided you give appropriate credit to the original author(s) and the source, provide a link to the Creative Commons license, and indicate if changes were made.

\section{References}

Andersen, S., Harrison, G.W., Lau, M.I., \& Rutström, E.E. (2008). Eliciting Risk and Time Preferences. Econometrica, 76(3), 583-618.

Andersen, S., Harrison, G.W., Lau, M.I., \& Rutström, E.E. (2014). Discounting Behavior: A Reconsideration. European Economic Review, 71, 15-33.

Barr, S., Shaw, G., \& Coles, T. (2011). Sustainable Lifestyles: Sites, Practices, and Policy. Environment and Planning A, 43(12), 3011-29.

Böhm, G., \& Pfister, H.-R. (2005). Consequences, Morality, and Time in Environmental Risk Evaluation. Journal of Risk Research, 8(6), 461-79.

Cairns, J., \& van der Pol, M. (1999). Do People Value Their Own Future Health Differently From Others' Future Health? Medical Decision Making, 19(4), 466-72.

Cairns, J.A. (1992). Health, Wealth and Time Preference. Project Appraisal, 7(1), 31-40.

Camerer, C.F., \& Hogarth, R.M. (1999). The Effects of Financial Incentives in Experiments: A Review and Capital-Labor-Production Framework. Journal of Risk and Uncertainty, 19(1-3), 7-42.

Chapman, G.B. (1996). Temporal Discounting and Utility for Health and Money. Journal of Experimental Psychology Learning Memory and Cognition, 22(3), 771-91.

Chapman, G.B., \& Elstein, A.S. (1995). Valuing the Future Temporal Discounting of Health and Money. Medical Decision Making, 15(4), 373-86.

Charness, G., Gneezy, U., \& Kuhn, M.A. (2012). Experimental Methods: Between-Subject and WithinSubject Design. Journal of Economic Behavior and Organization, 81(1), 1-8.

Coller, M., \& Williams, M.B. (1999). Eliciting Individual Discount Rates. Experimental Economics, 2(2), 107-27.

Cubitt, R.P., Starmer, C., \& Sugden, R. (1998). On the Validity of the Random Lottery Incentive System. Experimental Economics, 1(2), 115-31.

Cummings, R.G., Harrison, G.W., \& Rutström, E.E. (1995). Homegrown Values and Hypothetical Surveys: Is the Dichotomous Choice Approach Incentive-Compatible? American Economic Review, $85(1), 260-6$.

DEFRA (2008). A Framework for Pro-Environmental Behaviours. Technical report, Department for Environment Food and Rural Affairs.

Dohmen, T., Falk, A., Huffman, D., Sunde, U., Schupp, J., \& Wagner, G.G. (2011). Individual Risk Attitudes: Measurement, Determinants, and Behavioral Consequences. Journal of the European Economic Association, 9(3), 522-50.

Doyle, J.R. (2013). Survey of Time Preference, Delay Discounting Models. Judgment and Decision Making, 8(2), 116-35.

Eckel, C.C., \& Grossman, P.J. (2002). Sex Differences and Statistical Stereotyping in Attitudes Toward Financial Risk. Evolution and Human Behavior, 23(4), 281-95.

Eckel, C.C., \& Grossman, P.J. (2008). Forecasting Risk Attitudes: An Experimental Study Using Actual and Forecast Gamble Choices. Journal of Economic Behavior and Organization, 68(1), 1-17.

Einav, L., Finkelstein, A., Pascu, I., \& Cullen, M.R. (2012). How General Are Risk Preferences? Choices Under Uncertainty in Different Domains. American Economic Review, 102(6), 2606-38.

Ferecatu, A., \& Öncüler, A. (2016). Heterogeneous Risk and Time Preferences. Journal of Risk and Uncertainty, 53(1), in press.

Fischbacher, U. (2007). z-Tree: Zurich Toolbox for Ready-Made Economic Experiments. Experimental Economics, 10(2), 171-8.

Frederick, S. (2005). Cognitive Reflection and Decision Making. Journal of Economic Perspectives, 19(4), $25-42$.

Frederick, S., Loewenstein, G., \& O’Donoghue, T. (2002). Time Discounting and Time Preference: A Critical Review. Journal of Economic Literature, 40(2), 351-401. 
Gattig, A., \& Hendrickx, L. (2007). Judgmental Discounting and Environmental Risk Perception: Dimensional Similarities, Domain Differences, and Implications for Sustainability. Journal of Social Issues, 63(1), 21-39.

Green, L., Fry, A.F., \& Myerson, J. (1994). Discounting of Delayed Rewards: A Life-Span Comparison. Psychological Science, 5(1), 33-6.

Hardisty, D.J., Thompson, K.F., Krantz, D.H., \& Weber, E.U. (2013). How to Measure Time Preferences: An Experimental Comparison of Three Methods. Judgment and Decision Making, 8(3), 23649.

Hardisty, D.J., \& Weber, E.U. (2009). Discounting Future Green: Money Versus the Environment. Journal of Experimental Psychology: General, 138(3), 329-40.

Harrison, G.W., \& Lau, M.I. (2005). Is the Evidence for Hyperbolic Discounting in Humans Just an Experimental Artefact? Behavioral and Brain Sciences, 28, 657-7.

Hey, J.D., \& Lee, J. (2005). Do Subjects Separate (or Are They Sophisticated)? Experimental Economics, $8(3), 233-65$.

Holt, C.A., \& Laury, S.K. (2002). Risk Aversion and Incentive Effects. American Economic Review, 92(5), 1644-55.

Jesson, J. (2009). Household Waste Recycling Behavior: A Market Segmentation Model. Social Marketing Quarterly, 15(2), 25-38.

Kirby, K.N., \& Maraković, N.N. (1995). Modeling Myopic Decisions: Evidence for Hyperbolic DelayDiscounting within Subjects and Amounts. Organizational Behavior and Human Decision Processes, 64(1), 22-30.

Kroll, Y., Levy, H., \& Rapoport, A. (1988). Experimental Tests of the Separation Theorem and the Capital Asset Pricing Model. American Economic Review, 78(3), 500-19.

Laury, S.K. (2005). Pay One or Pay All: Random Selection of One Choice for Payment. Discussion Paper, Andrew Young School, Georgia State University.

Madden, G.J., Bickel, W.K., \& Jacobs, E.A. (1999). Discounting of Delayed Rewards in OpioidDependent Outpatients: Exponential or Hyperbolic Discounting Functions? Experimental and Clinical Psychopharmacology, 7(3), 284-93.

Nordhaus, W.D. (2007). A Review of the Stern Review on the Economics of Climate Change. Journal of Economic Literature, 45(3), 686-702.

Raineri, A., \& Rachlin, H. (1993). The Effect of Temporal Constraints on the Value of Money and Other Commodities. Journal of Behavioral Decision Making, 6(2), 77-94.

Read, D., Frederick, S., Orsel, B., \& Rahman, J. (2005). Four Score and Seven Years from Now: The Date/Delay Effect in Temporal Discounting. Management Science, 51(9), 1326-35.

Riddel, M. (2012). Comparing Risk Preferences over Financial and Environmental Lotteries. Journal of Risk and Uncertainty, 45(2), 135-57.

Stern, N. (2007). The Economics of Climate Change: The Stern Review: Technical report, Cambridge University Press.

Tsukayama, E., \& Duckworth, A.L. (2010). Domain-Specific Temporal Discounting and Temptation. Judgment and Decision Making, 5(2), 72-82.

Ubfal, D. (2016). How General are Time Preferences? Eliciting Good-Specific Discount Rates. Journal of Development Economics, 118, 150-70.

Viscusi, W.K., Huber, J., \& Bell, J. (2008). Estimating Discount Rates for Environmental Quality from Utility-Based Choice Experiments. Journal of Risk and Uncertainty, 37(2-3), 199-220.

Weber, E.U., Blais, A., \& Betz, N.E. (2002). A Domain-Specific Risk-Attitude Scale: Measuring Risk Perceptions and Risk Behaviors. Journal of Behavioral Decision Making, 15(4), 263-90.

Weitzman, M.L. (2007). A Review of the Stern Review on the Economics of Climate Change. Journal of Economic Literature, 45(3), 703-24. 